\title{
Narcolepsy Following Yellow Fever Vaccination: A Case Report
}

\author{
Richard E. Rosch ${ }^{1,2 *}$, Michael Farquhar ${ }^{3}$, Paul Gringras ${ }^{3}$ and Deb K. Pal4,5 \\ ${ }^{1}$ Wellcome Trust Centre for Neuroimaging, Institute of Neurology, University College London, London, UK, ${ }^{2}$ Centre for \\ Developmental Cognitive Neuroscience, Institute of Child Health, University College London, London, UK, ${ }^{3}$ Children's Sleep \\ Medicine Department, Evelina London Children's Hospital, Guy's and St. Thomas' NHS Foundation Trust, London, UK, \\ ${ }^{4}$ Department of Basic and Clinical Neurosciences, Institute of Psychiatry, Psychology and Neuroscience, King's College \\ London, London, UK, ${ }^{5}$ Department of Paediatric Neurology, Evelina London Children's Hospital, Guy's and St. Thomas' \\ NHS Foundation Trust, London, UK
}

\section{OPEN ACCESS}

Edited by:

Ahmed S. BaHammam, King Saud University, Saudi Arabia

Reviewed by: Thomas Penzel, Charité Universitätsmedizin Berlin, Germany Fang Han,

Peking University People Hospital, China

*Correspondence: Richard E. Rosch r.rosch@ucl.ac.uk

Specialty section:

This article was submitted to Sleep and Chronobiology,

a section of the journal

Frontiers in Neurology

Received: 17 June 2016

Accepted: 27 July 2016

Published: 10 August 2016

Citation:

Rosch RE, Farquhar $M$, Gringras $P$ and Pal DK (2016) Narcolepsy

Following Yellow Fever

Vaccination: A Case Report.

Front. Neurol. 7:130.

doi: 10.3389/fneur.2016.00130
Narcolepsy with cataplexy is a rare, but important differential diagnosis for daytime sleepiness and atonic paroxysms in an adolescent. A recent increase in incidence in the pediatric age group probably linked to the use of the Pandemrix influenza vaccine in 2009, has increased awareness that different environmental factors can "trigger" narcolepsy with cataplexy in a genetically susceptible population. Here, we describe the case of a 13-year-old boy with narcolepsy following yellow fever vaccination. He carries the HLA DQB1*0602 haplotype strongly associated with narcolepsy and cataplexy. Polysomnography showed rapid sleep onset with rapid eye movement (REM) latency of $47 \mathrm{~min}$, significant sleep fragmentation and a mean sleep latency of 1.6 min with sleep onset REM in four out of four nap periods. Together with the clinical history, these findings are diagnostic of narcolepsy type 1 . The envelope protein $E$ of the yellow fever vaccine strain 17D has significant amino acid sequence overlap with both hypocretin and the hypocretin receptor 2 receptors in protein regions that are predicted to act as epitopes for antibody production. These findings raise the question whether the yellow fever vaccine strain may, through a potential molecular mimicry mechanism, be another infectious trigger for this neuro-immunological disorder.

Keywords: sleep disorders, molecular immunogene, HLA, pediatric neurology

\section{INTRODUCTION}

Narcolepsy is a rare, lifelong sleep disorder that is usually diagnosed in adolescence and early adulthood. It is classified as narcolepsy type 1 vs. narcolepsy type 2 according to the presence or absence of cataplexy and cerebrospinal fluid (CSF) hypocretin deficiency (1). While the exact etiology is unclear, narcolepsy with cataplexy is strongly associated with a significant loss of hypocretin-secreting neurons in the lateral hypothalamus alongside reduced CSF hypocretin-1 levels. Furthermore, there is a well-documented association with HLA DQB1 ${ }^{\star} 0602$, suggesting a possible autoimmune etiology (2). Here, we present a patient from our tertiary pediatric clinical service, who has a clear history of narcolepsy with cataplexy following recent yellow fever vaccination.

A recent increase in reported cases of narcolepsy with cataplexy has been associated both with the use of the ASO3-conjugated split-virion H1N1 vaccine Pandemrix in 2009 (3), and with H1N1 infection (4). Influenza nucleoprotein antibodies have been shown to cross-react with hypocretin receptors (HCRTR1, HCRTR2), suggesting a form of molecular mimicry as the cause for the hypocretin neuron loss (5). Most patients with this "post-vaccination" narcolepsy develop symptoms at 
a younger than usual age with abrupt onset of cataplexy shortly after the vaccination (mean interval 7 weeks), resulting in an increase of narcolepsy incidence in the pediatric age group in recent years.

\section{CASE REPORT}

A 13-year-old boy attended pediatric neurology clinic with a 2-year history of repeated paroxysmal muscle tone loss, in order to be assessed for possible atonic seizures. Having been previously neurologically intact, symptoms started with an episode in a restaurant where he abruptly lost axial tone and his head hit the table. He subsequently experienced frequent similar episodes, especially when experiencing positive emotions, during laughter or while playing games with excitement. Episodes were varied as follows: they ranged from subtle involuntary head nods, which he could conceal and were not always noticed by others, to complete postural collapse. They were consistently symmetrical, involving both the left and right side of his body. All instances were described as short-lived, lasting no longer than a few seconds.

During the same period, he would drift off to sleep several times a day without warning, and not wake up until roused. At night, his sleep was interrupted, waking up frightened while feeling unable to move. In addition, his mother also noticed a marked weight gain associated with a significant increase in his appetite. He was previously well, with an unremarkable birth and family history. He never received the Pandemrix influenza vaccine, but $\sim 2$ weeks prior to symptom onset had been vaccinated with the Stamaril live, attenuated yellow fever vaccine for a family trip to Africa.

Neurological clinical examination was unremarkable and initial investigations, including electrocardiography (ECG), brain magnetic resonance imaging (MRI), and routine blood tests were within normal limits. Polysomnography (PSG) showed rapid sleep onset with rapid eye movement (REM) latency of $47 \mathrm{~min}$ (normal 70-110, full results given in Table 1, hypnogram of overnight sleep and multiple sleep latency is shown in Figure 1). He had significant sleep fragmentation, with frequent REM intrusions interrupting the usual consolidation of non-REM periods but no evidence of sleep disordered breathing. Multiple sleep latency test (MSLT) performed the following day was significantly abnormal with a mean sleep latency of $1.6 \mathrm{~min}$ (normal $>8 \mathrm{~min}$ ) and sleep onset REM evident in four out of four nap periods.

The clinical history of weight gain, hypnagogic hallucinations, sleep paralysis, and clear episodes of loss of muscle tone (cataplexy) is strongly suggestive of narcolepsy. With that clinical context, the fragmented sleep without sleep disordered breathing on PSG together with significantly shortened sleep latency and sleep onset REM on MSLT confirmed the diagnosis of narcolepsy. Periods of non-REM sleep were interrupted by REM intrusions, resulting in a proportional increase in REM sleep compared to non-REM sleep phases (as indicated in Table 1). While sleep fragmentation does occur for other reasons, this pattern shown on PSG is typical of young patients with untreated narcolepsy. These findings taken together meet level 2 evidence for narcolepsy according to the criteria laid out by the Brighton Collaboration Narcolepsy
TABLE 1 | Polysomnography results of the patient at time of diagnosis.

\begin{tabular}{llc}
\hline Measurement & Value & Reference \\
\hline Nocturnal polysomnography & & - \\
Total sleep time, min & 646.6 & $23(\mathrm{SD}=25)$ \\
Sleep onset latency, min & 0.7 (rapid) & $88(\mathrm{SD}=41)$ \\
REM latency, min & 47 (rapid) & $9.3(\mathrm{SD}=4.8)$ \\
Arousal index, per hour & 16.4 (slightly elevated) & $89(\mathrm{SD}=7.5)$ \\
Sleep efficiency & $82.5 \%$ (slightly low) & $>96$ \\
Oxygen saturation, \% & 98.1 & $1(\mathrm{SD}=0.8)$ \\
Apnea hypopnea index, per hour & 1.5 & $\sim 6^{\mathrm{b}}$ \\
Sleep stages, \% of total sleep time & $\sim 49^{\mathrm{b}}$ \\
N1 & 5.9 & $\sim 23^{\mathrm{b}}$ \\
N2 & 41.1 & $\sim 22^{\mathrm{b}}$ \\
N3 & 18.5 & \\
REM & 34.5 & $>8^{\mathrm{a}}$ \\
Multiple sleep latency test & & $0^{\mathrm{a}}$ \\
Mean sleep latency test, min & 1.6 (rapid) & \\
Sleep onset REM periods & 4 of 4 nap periods & \\
& (suggestive of narcolepsy) &
\end{tabular}

alndicates significantly abnormal results.

${ }^{b}$ Reference values from Ref. (6) given for comparison, no confidence intervals given. $R E M$, rapid eye movement.

Working Group (7), as well as fulfilling the diagnostic criteria for narcolepsy type 1 defined in the International Classification of Sleep Disorders, third edition (1).

He was found to have the strongly associated HLA-type $\mathrm{DQB} 1{ }^{*} 0602$. MRI brain, including T1 and T2 weighted scan and FLAIR imaging was normal, with normal hypothalamic appearances. Because of the clear clinical history of cataplexy and the invasiveness of the procedure in a pediatric patient, CSF levels of hypocretin (orexin) were not measured. Treatment was mainly directed at managing daytime sleepiness through improved sleep hygiene and daytime naps, in addition to stimulant medication (slow release methylphenidate, $18 \mathrm{mg}$ once a day increased in two steps to $45 \mathrm{mg}$ once a day) and anti-cataplectic medication (venlafaxine, $37.5 \mathrm{mg}$ once a day).

\section{DISCUSSION}

The case presented here raises the possibility that currently unknown combinations of genetic susceptibilities and environmental triggers may play a role in the etiology of narcolepsy in some patients. Our patient never received Pandemrix, while having received the yellow fever vaccine Stamaril 2 weeks prior to cataplexy onset. In cases reported in association with Pandemrix, the risk of developing narcolepsy was most increased in this early post-vaccination time period (8-42 days) (8). The yellow fever vaccines induce a strong immune response, and the YFV-17D strain used in the Stamaril vaccines contains multiple antigens with several epitopes each that can bind different HLA molecules (9). While yellow-fever-vaccine-associated neurological disease is described, narcolepsy is not currently a recognized complication (10).

Previous studies have shown that the association with HLA DQB $1^{\star} 0602$ is extremely high in patients with typical or severe cataplexy [85-95\% of patients show this HLA type vs. $25 \%$ of age-matched controls (11)]. Together with epidemiological 


\section{A Nocturnal Polysomnography (Day 1)}

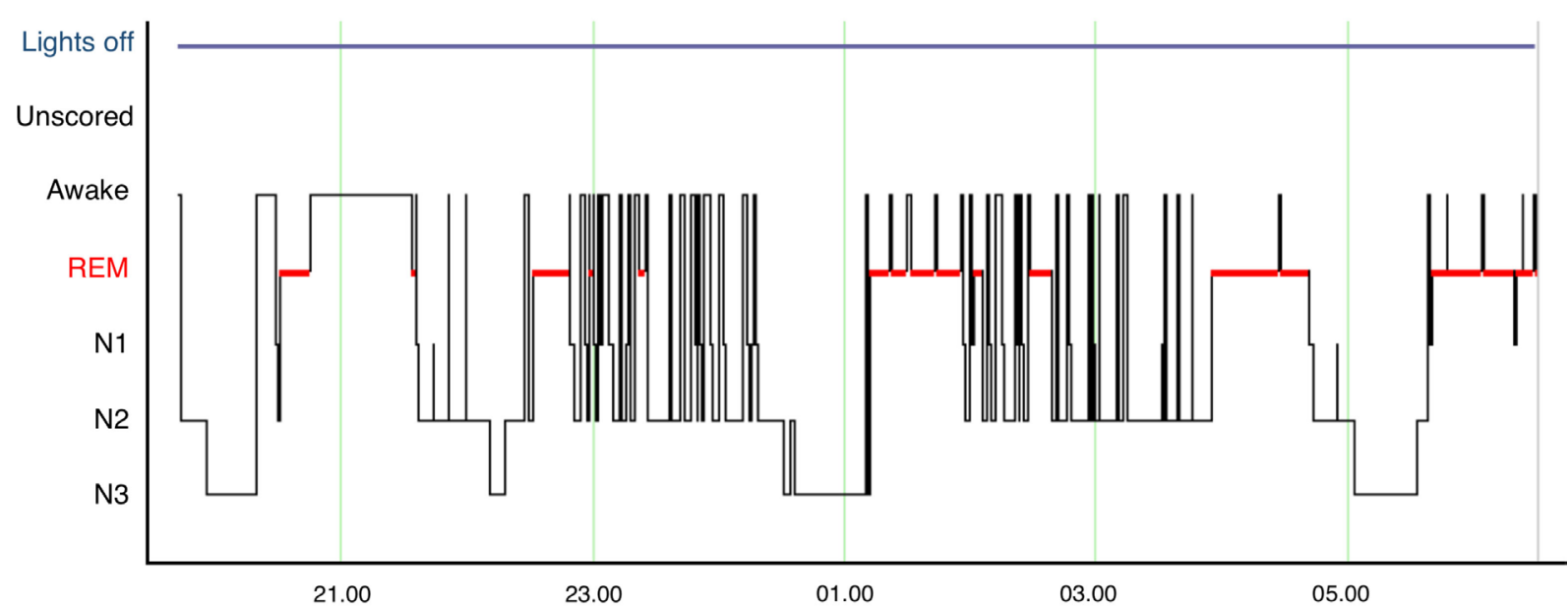

B $\quad$ Multiple Sleep Latency Test (Day 2)

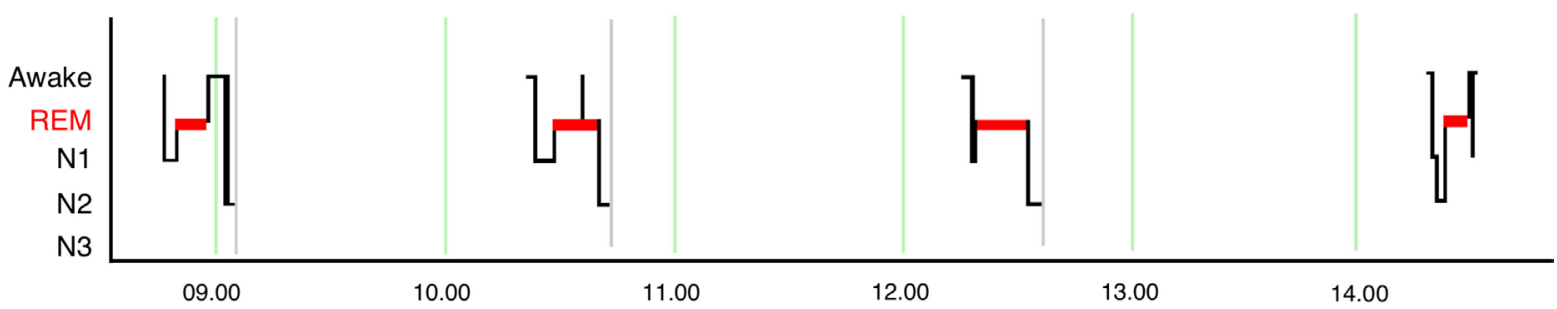

FIGURE 1 | (A) Hypnogram of nocturnal polysomnography for patient showing significant sleep fragmentation with frequent arousals and awakenings, particularly during N2 sleep. The image also shows the short sleep latency and frequent sleep onset REM episodes after awakenings. (B) Hypnogram of multiple sleep latency showing sleep onset REM in 4 of 4 nap periods.

associations between narcolepsy onset and different environmental triggers [including Streptococcus sp. Infection (12), H1N1 infection (13), and the Pandemrix vaccine (7)], an immunemediated, environmentally triggered etiology appears most likely (14). Most recently, different lines of evidence have suggested that the H1N1- and Pandemrix-associated cases of narcolepsy are caused by molecular mimicry between influenza epitopes and hypocretin receptor domains with associated antibody-mediated destruction of hypocretin-secreting neurons (5).

The attenuated yellow fever strain used in the Stamaril vaccine (YFV-17D) carries a genome that codes for 13 distinct proteins and peptides [UniProt ID P03314 (15)]. Amino acid sequences for each of the 17D strain peptides and proteins were assessed for sequence overlap with hypocretin, HCRTR1, and HCRTR2 using the BLAST algorithm (see blast.ncbi.nlm.nih.gov for more information). Of these, four show significant amino acid sequence similarities to hypocretin or one of its receptors (Table 2).

Sequence overlaps in extracellular domains of the human proteins are of particular interest as these are the most plausible target for putative antibody-mediated molecular mimicry. Only one of the viral peptides (the envelope protein E) has sequence overlaps with extracellular protein components (i.e., hypocretin, or external domains of the hypocretin receptors).
Sections within the same protein sequence are also predicted to act as epitopes for B-cell linear proliferation (Figure 2). These findings taken together illustrate one specific possible mechanism for molecular mimicry between the yellow fever vaccine strain, and hypocretin-secreting neurons: antibodies are produced to epitopes found in the envelope protein $\mathrm{E}$ sequence as part of the strong immune response to the vaccine. These may then cross-react with either hypocretin molecules themselves, or an extracellular domain of the hypocretin receptor 2 , because of the amino acid sequence similarities between the vaccine epitopes and the hypocretin or receptor molecules.

Clearly, our single case does not allow any inference whether Stamaril has causally contributed to the development of narcolepsy in this patient. Hypocretin-associated autoantibodies, where detected at all, do not seem to be more common in patients with narcolepsy compared to controls $(18,19)$. And even for the Pandemrix vaccine, where there is strong epidemiological evidence for a causative link, there is conflicting evidence as to whether molecular mimicry plays a role in the pathophysiology $(5,20)$.

Yet our case raises the consideration as to whether other associations between environmental triggers and narcolepsy onset exist, particularly in the context of the genetic susceptibility conferred by the associated HLA type. 
TABLE 2 | Sequence overlap between YFV-D17 proteins and hypocretin and its receptors.

\begin{tabular}{|c|c|c|c|c|c|}
\hline Protein/peptide & AA\# & Reference sequence & AA\# & Cellular location & E-value \\
\hline Capsid protein C & $2-13$ & Hypocretin receptor 1 & $157-168$ & Cytoplasmic/transmemrbrane & 0.14 \\
\hline Capsid protein C & $65-74$ & Hypocretin receptor 2 & $262-271$ & Cytoplasmic & 0.29 \\
\hline Envelope protein $\mathrm{E}$ & 224-235 & Hypocretin receptor 2 & $43-54$ & Extracellular & 0.47 \\
\hline Envelope protein $\mathrm{E}$ & $218-253$ & Hypocretin & $70-103$ & Extracellular & 0.055 \\
\hline Non-structural protein 2A-alpha & $128-152$ & Hypocretin receptor 2 & $137-161$ & Cytoplasmic/transmembrane & 0.63 \\
\hline Serine protease subunit NS2B & $95-108$ & Hypocretin receptor 2 & $422-435$ & Cytoplasmic & 0.11 \\
\hline
\end{tabular}

AA\#, position in the amino acid sequence (start-stop); E-value, "Expect" value is a parameter that describes the number of hits "expected" chance results; the lower the score, the more "significant" the match is (see http://blast.ncbi.n/m.nih.gov/ for more information).

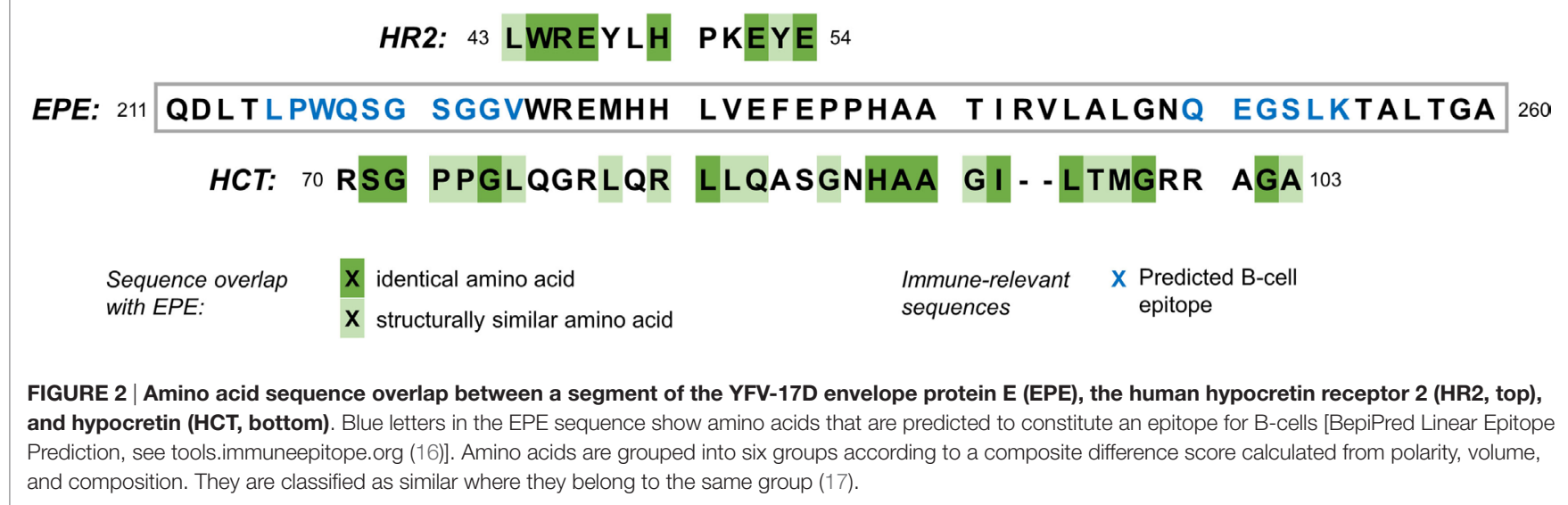

\section{CONCLUSION}

Narcolepsy with cataplexy in children is strongly associated with HLA DQB1 ${ }^{\star} 0602$ and a loss of hypothalamic hypocretinsecreting neurons. There is epidemiological evidence that a recent increase in narcolepsy diagnoses in children was linked to the H1N1 mass vaccination.

The close temporal association of the live-attenuated yellow fever vaccine, associated with genetic susceptibility, and the potential for molecular mimicry between MHC-derived peptides, hypocretin, its receptors, and the viral envelope protein $\mathrm{E}$ raises the question as to whether the vaccine was involved in the pathology here. Exploring any such association will need further careful evaluation using epidemiological approaches. The next step for this will be a detailed evaluation of the incidence of narcolepsy in possible at risk population (i.e., patients exposed to yellow fever in endemic regions, young people undergoing yellow fever vaccine). As with the Pandemrix vaccine-related cases, further longitudinal follow-up is necessary, as it is not yet clear whether presumed vaccine-associated cases follow the same natural history as seen in other narcolepsy patients.

\section{ETHICS STATEMENT}

No investigations or interventions were performed outside routine clinical care for this patient. As this is a case report, without experimental intervention into routine care, no formal research ethics approval was required; Written, fully informed consent was given and recorded from the patient's parents. Verbal assent was given by the (underage) patient himself; this case study reports routine clinical care provided for a paediatric patient only.

\section{AUTHOR CONTRIBUTIONS}

Dr. MF and Prof. DP were involved in the work-up of the patient, planning and conducting investigations, and providing clinical care. They reviewed and revised the manuscript and approved the final manuscript as submitted. Dr. RR planned the case report, drafted the initial manuscript, reviewed and revised the manuscript, and approved the final manuscript as submitted. Prof. PG helped planning clinical investigations, critically reviewed the manuscript, implemented substantial changes to the argument, and approved the final manuscript as submitted.

\section{ACKNOWLEDGMENTS}

We are grateful to the patient and his family to participate in this case report, to Ms Laura Crewe for her help in preparing the images for publication and to Prof. Harold Baum for his advice on interpreting the sequence homologies. Dr. RR is supported by the Wellcome Trust (106556/Z/14/Z). 


\section{REFERENCES}

1. American Academy of Sleep Medicine. International Classification of Sleep Disorders. 3rd ed. Darien, IL: American Academy of Sleep Medicine (2014).

2. Mahlios J, De la Herrán-Arita AK, Mignot E. The autoimmune basis of narcolepsy. Curr Opin Neurobiol (2013) 23:767-73. doi:10.1016/j.conb.2013.04.013

3. Thebault S, Vincent A, Gringras P. Narcolepsy and H1N1 vaccination: a link? Curr Opin Pulm Med (2013) 19:587-93. doi:10.1097/MCP.0b013e328365af97

4. Han F, Lin L, Warby SC, Faraco J, Li J, Dong SX, et al. Narcolepsy onset is seasonal and increased following the $2009 \mathrm{H} 1 \mathrm{~N} 1$ pandemic in China. Ann Neurol (2011) 70:410-7. doi:10.1002/ana.22587

5. Ahmed SS, Volkmuth W, Duca J, Corti L, Pallaoro M, Pezzicoli A, et al. Antibodies to influenza nucleoprotein cross-react with human hypocretin receptor 2. Sci Transl Med (2015) 7:294ra105. doi:10.1126/scitranslmed. aab2354

6. Ohayon MM, Carskadon MA, Guilleminault C, Vitiello MV. Meta-analysis of quantitative sleep parameters from childhood to old age in healthy individuals: developing normative sleep values across the human lifespan. Sleep (2004) 27:1255-73.

7. Poli F, Overeem S, Lammers GJ, Plazzi G, Lecendreux M, Bassetti CL, et al. Narcolepsy as an adverse event following immunization: case definition and guidelines for data collection, analysis and presentation. Vaccine (2013) 31:994-1007. doi:10.1016/j.vaccine.2012.12.014

8. European Centre for Disease Prevention and Control. Narcolepsy in Association with Pandemic Influenza Vaccination. A Multi-Country European Epidemiological Investigation. Stockholm: ECDC (2012).

9. de Melo AB, Nascimento EJ, Braga-Neto U, Dhalia R, Silva AM, Oelke M, et al. T-cell memory responses elicited by yellow fever vaccine are targeted to overlapping epitopes containing multiple HLA-I and -II binding motifs. PLoS Negl Trop Dis (2013) 7:e1938. doi:10.1371/journal.pntd.0001938

10. Breugelmans JG, Lewis RF, Agbenu E, Veit O, Jackson D, Domingo C, et al. Adverse events following yellow fever preventive vaccination campaigns in eight African countries from 2007 to 2010. Vaccine (2013) 31:1819-29. doi:10.1016/j.vaccine.2013.01.054

11. Mignot E, Hayduk R, Black J, Grumet FC, Guilleminault C. HLA DQB1*0602 is associated with cataplexy in 509 narcoleptic patients. Sleep (1997) 20:1012-20.
12. Aran A, Lin L, Nevsimalova S, Plazzi G, Hong SC, Weiner K, et al. Elevated anti-streptococcal antibodies in patients with recent narcolepsy onset. Sleep (2009) 32:979-83. doi:10.1016/S0513-5117(10)79188-2

13. Han F, Lin L, Li J, Dong XS, Mignot E. Decreased incidence of childhood narcolepsy 2 years after the $2009 \mathrm{H} 1 \mathrm{~N} 1$ winter flu pandemic. Ann Neurol (2013) 73:560. doi:10.1002/ana.23799

14. Liblau RS, Vassalli A, Seifinejad A, Tafti M. Hypocretin (orexin) biology and the pathophysiology of narcolepsy with cataplexy. Lancet Neurol (2015) 14:318-28. doi:10.1016/S1474-4422(14)70218-2

15. The UniProt Consortium. UniProt: a hub for protein information. Nucleic Acids Res (2014) 43:D204-12. doi:10.1093/nar/gku989

16. Larsen JEP, Lund O, Nielsen M. Improved method for predicting linear B-cell epitopes. Immunome Res (2006) 2:2. doi:10.1186/1745-7580-2-2

17. Miyata T, Miyazawa S, Yasunaga T. Two types of amino acid substitutions in protein evolution. J Mol Evol (1979) 12:219-36. doi:10.1007/ BF01732340

18. Tanaka S, Honda Y, Inoue Y, Honda M. Detection of autoantibodies against hypocretin, hcrtrl, and hcrtr2 in narcolepsy: anti-Hcrt system antibody in narcolepsy. Sleep (2006) 29:633-8.

19. Black JL 3rd, Silber MH, Krahn LE, Fredrickson PA, Pankratz VS, Avula R, et al. Analysis of hypocretin (orexin) antibodies in patients with narcolepsy. Sleep (2005) 28:427-31.

20. Vassalli A, Li S, Tafti M. Comment on 'Antibodies to influenza nucleoprotein cross-react with human hypocretin receptor 2'. Sci Transl Med (2015) 7:314le2. doi:10.1126/scitranslmed.aad2353

Conflict of Interest Statement: The authors declare that the research was conducted in the absence of any commercial or financial relationships that could be construed as a potential conflict of interest.

Copyright (C) 2016 Rosch, Farquhar, Gringras and Pal. This is an open-access article distributed under the terms of the Creative Commons Attribution License (CC BY). The use, distribution or reproduction in other forums is permitted, provided the original author(s) or licensor are credited and that the original publication in this journal is cited, in accordance with accepted academic practice. No use, distribution or reproduction is permitted which does not comply with these terms. 